\title{
Estudo comparativo da intoxicação experimental por amitraz entre cães e gatos
}

Silvia Franco ANDRADE ${ }^{1}$

Cecília Braga LAPOSY ${ }^{1}$

Luciana Teramossi

RODRIGUES ${ }^{1}$

Jacqueline MARCICANO

Carlos Vianna de ANDRADE JR ${ }^{1}$

Thiago Luiz APPEL ${ }^{1}$

Correspondência para:

Silvia Franco Andrade-Curso de Medicina Veterinária - UNOESTE, Campus II, Rodovia Raposo Tavares, Km 572 CEP 19067-175, Presidente Prudente, SP, silviafranco@unoeste.br

Recebido para publicação: 11/12/2006 Aprovado para publicação: 21/09/2007

\author{
1 - Curso de Medicina Veterinária da Universidade do Oeste Paulista, \\ Presidente Prudente - SP
}

\section{Resumo}

O objetivo do presente trabalho foi estudar as características em comum e as diferenças observadas na intoxicação induzida por $1 \mathrm{mg} / \mathrm{kg}$ de amitraz, IV, em cães e gatos. Os principais sinais clínicos observados em comum foram sedação, hipotermia, bradicardia, bradiarritmias, hipotensão, bradipnéia, midríase e hiperglicemia transitória, porém a intensidade destes sinais foi diferente entre as espécies. A hipotermia foi mais acentuada em gatos. Os cães foram mais sensíveis às alterações cardiorespiratórias apresentando diminuição mais significativa na freqüência cardíaca e respiratória, além de ocorrência de maior número de bradiarritmias. Os gatos apresentaram midríase mais prolongada do que os cães. Observou-se hiperglicemia e hipoinsulinemia transitórias e diminuição transitória dos níveis plasmáticos de cortisol em ambas espécies, porém os gatos apresentaram um pico de hiperglicemia maior e mais precoce do que os cães, e com relação aos níveis plasmáticos de cortisol, os cães apresentaram uma diminuição mais acentuada do que os gatos. O tempo médio de retorno da sedação foi mais prolongado em gatos. Estes resultados mostraram que a intoxicação por amitraz entre cães e gatos é muito similar, porém os gatos demonstraram maior sensibilidade à indução de hipotermia e hiperglicemia, além de midríase e um tempo médio de retorno da sedação mais prolongado, enquanto os cães apresentaram diminuição mais acentuada nos parâmetros cardiorespiratórios e dos níveis plasmáticos de cortisol do que os gatos.

\section{Introdução}

O amitraz é um antiparasitário do grupo das formamidinas, que é utilizado como acaricida e carrapaticida em Medicina Veterinária. O produto é relativamente barato e de fácil aquisição, o que torna o seu uso bastante popular no Brasil. ${ }^{1} \mathrm{O}$ seu mecanismo de ação consiste na inibição da enzima monoaminoxidase (MAO) e, principalmente, como um agonista em $\alpha_{2}-$ adreneceptores. $^{2}$

Os sinais clínicos e as alterações laboratoriais induzidas pela intoxicação por amitraz são caracterizados por sedação, perda de reflexos, letargia, bradicardia, bradiarritmias, hipotensão, hipotermia, hiperglicemia, poliúria, bradipnéia, midríase, vômito, salivação e impactação intestinal.,34
Em cães, o amitraz é utilizado topicamente para o tratamento de escabiose e demodicose ${ }^{2,5}$, e no controle de carrapatos ${ }^{1}$. Em gatos é eficiente no tratamento de escabiose e demodicose felinas. ${ }^{6,7} \mathrm{Em}$ um estudo retrospectivo sobre as causas mais comuns das intoxicações em cães e gatos atendidos no Hospital Veterinário da Universidade de São Paulo, entre 1998 a 2000 , observou-se que em cães $25 \%$ dos casos de intoxicação eram por amitraz. ${ }^{8}$ Em gatos, há alguns casos relatados desta intoxicação nesta espécie. ${ }^{4,9,10}$

Apesar de ser uma intoxicação bem documentada em cães s,11,12,13 $^{3,}$ recentemente estudada em gatos ${ }^{14}$, não há estudos comparativos entre essas duas espécies, o que contribuiria com novas informações para a Toxicologia Veterinária, sendo o objetivo 
deste trabalho.

\section{Material e Método}

O experimento foi aprovado pelo Comitê de Ética em Pesquisa da Universidade do Oeste Paulista (UNOESTE) protocolo $\mathrm{n}^{0}$ 086/03. O experimento foi realizado no Hospital Veterinário da UNOESTE - Presidente Prudente - SP.

Foram utilizados oito cães e oito gatos, sem raça definida (SRD); cães com idade entre 1 a 5 anos, com peso variando de

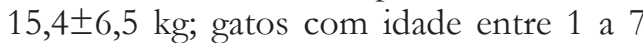
anos, peso variando de $3,6 \pm 0,6$, provenientes do canil e gatil Central da UNOESTE. Os animais foram previamente selecionados 24 horas antes do experimento por meio da normalidade dos exames clínico e laboratorial, eritrograma, leucograma, uréia, creatinina, ALT e AST. Os animais que não se apresentaram em boas condições foram descartados do experimento. Os cães e gatos foram mantidos em gaiolas individuais em um ambulatório de experimentação 24 horas antes do experimento se iniciar e mantidos até seu pronto restabelecimento após a intoxicação.A alimentação foi à base de ração comercial seca para cães e gatos à vontade e água também à vontade.

A administração de amitraz foi feita da seguinte forma:

- cães: administrou-se amitraz (Mitrax ${ }^{\circledR}$, Agribrands Purina, Paulínia, Brasil) na dose de $1 \mathrm{mg} / \mathrm{kg} \mathrm{IV}$, preparado a 2,5\% (25 mg/mL), diluindo-se $1 \mathrm{~mL}$ de amitraz em $4 \mathrm{~mL}$ de água bidestilada. A dose e a diluição foram baseadas no modelo experimental descrito para cães ${ }^{12}$.

- gatos: administrou-se amitraz na dose de $1 \mathrm{mg} / \mathrm{kg}$ IV, preparado a 1,5\% (15 $\mathrm{mg} / \mathrm{ml}$ ), diluindo-se $0,6 \mathrm{~mL}$ de amitraz em 4,4 mL de água bidestilada. A dose e a diluição foram baseadas no modelo experimental descrito para gatos ${ }^{14}$.

Foram avaliados os seguintes parâmetros: temperatura retal $(\mathrm{T})$ em graus Celsius $\left({ }^{\circ} \mathrm{C}\right)$; freqüência cardíaca (FC) em batimentos por minuto (bpm); eletrocardiograma (ECG); pressão arterial sistólica (PAS) em mmHg pelo método Doppler; freqüência respiratória (FR) em movimentos por minuto ( $\mathrm{mpm})$; diâmetro pupilar (DP) avaliado através do teste pupilar com luz puntiforme direcionada para a pupila e graduado em: (1) normal, (2) midríase, (3) miose. Os momentos da avaliação foram: $0 \mathrm{~min}$ (T0), $30 \mathrm{~min}$ (T30), $60 \mathrm{~min}$ (T60), $120 \mathrm{~min}$ (T120), $180 \mathrm{~min}$ (T180), $240 \mathrm{~min}$ (T240) e $360 \mathrm{~min}$ (T360) após a intoxicação por amitraz. Foram coletadas amostras de sangue nos tempos T0, T60, T180 e T180 para realização de dosagem de glicose, insulina e cortisol. A dosagem de glicose foi realizada pelo método enzimático colorimétrico por espectrofotômetro no Laboratório Clínico do HV da UNOESTE. As dosagens de insulina e cortisol foram realizadas pelo método de radioimunoensaio no Laboratório Central de Radioimunoensaio de São Paulo - CRIESP.

O tempo médio de recuperação da sedação (TMRS) foi considerado como o tempo gasto, em minutos, para o animal se levantar após a administração do amitraz e após a administração do antagonista $\alpha_{2}$ adrenérgico, ioimbina ou atipamezole.

Foi utilizada para cada variável a análise de variância pelo teste $F^{15}$. Foi adotado o nível de significância de 5\%.

\section{Resultados e Discussão}

As alterações observadas na temperatura corpórea estão descritas na tabela 1. Os gatos foram mais sensíveis à hipotermia induzida pelo amitraz havendo uma redução de 4,7\% ao final do experimento (T360), enquanto essa redução foi de $2,4 \%$ em cães. Este efeito pode ser atribuído à atuação do amitraz como agonista a-adrenérgico afetando o centro de termorregulação localizado no hipotálamo. ${ }^{2}$ Os gatos, em particular, são bastante susceptíveis à hipotermia em algumas situações, como na recuperação anestésica com o uso de agonistas $\alpha_{2}$-adrenérgicos. ${ }^{16}$

A sensibilidade às alterações 
Tabela 1 - Médias e desvios padrões das variáveis: temperatura (T), freqüência cardíaca (FC), pressão arterial sistólica (PAS), freqüência respiratória (FR) e. Medianas e percentis (P25; P75) da variável: diâmetro pupilar (DP) (1 - normal; 2 - midríase; 3 - miose) em cães e gatos intoxicados por amitraz $(1 \mathrm{mg} / \mathrm{kg}, \mathrm{IV})$

\begin{tabular}{|c|c|c|c|c|c|c|c|c|}
\hline \multirow{2}{*}{ Variável } & \multirow{2}{*}{ Animal } & \multicolumn{7}{|c|}{ TEMPO (min) } \\
\hline & & T0 & T30 & T60 & T120 & T180 & $\mathrm{T} 240$ & T360 \\
\hline \multirow{2}{*}{$\mathrm{T}$} & Cão & $38,9 \pm 0,2$ & $\begin{array}{c}38,4 \pm 0,2 \\
(\downarrow 1,7 \%)\end{array}$ & $\begin{array}{c}37,7 \pm 0,9 * \\
(\downarrow 3,1 \%)\end{array}$ & $\begin{array}{c}36,7 \pm 0,9 * \\
(\downarrow 5,7 \%)\end{array}$ & $\begin{array}{c}36,9 \pm 0,9 * \\
(\downarrow 5,8 \%)\end{array}$ & $\begin{array}{c}37,6 \pm 0,8^{*} \\
(\downarrow 3,4 \%)\end{array}$ & $\begin{array}{l}38,0 \pm 0,9 \\
(\downarrow 2,4 \%)\end{array}$ \\
\hline & Gato & $38,5 \pm 0,7$ & $\begin{array}{c}38,0 \pm 0,8^{*} \\
(\downarrow 1,3 \%)\end{array}$ & $\begin{array}{c}37,5 \pm 0,6^{*} \\
(\downarrow 2,6 \%)\end{array}$ & $\begin{array}{c}36,6 \pm 0,7^{*} \\
(\downarrow 5,0 \%)\end{array}$ & $\begin{array}{c}36,5 \pm 1,3^{*} \\
(\downarrow 5,2 \%)\end{array}$ & $\begin{array}{c}36,8 \pm 1,7^{*} \\
(\downarrow 4,5 \%)\end{array}$ & $\begin{array}{c}36,7 \pm 1,4^{*} \\
(\downarrow 4,7 \%)\end{array}$ \\
\hline \multirow{2}{*}{$\mathrm{FC}$} & Cão & $122,5 \pm 21,6$ & $\begin{array}{c}59,5 \pm 7,8^{*} \\
(\downarrow 51,5 \%)\end{array}$ & $\begin{array}{c}57,0 \pm 10,8^{*} \\
(\downarrow 53,5 \%)\end{array}$ & $\begin{array}{c}51,6 \pm 7,8^{*} \\
(\downarrow 57,9 \%)\end{array}$ & $\begin{array}{c}63,6 \pm 21,2^{*} \\
(\downarrow 48,5 \%)\end{array}$ & $\begin{array}{c}82,8 \pm 15,5^{*} \\
(\downarrow 32,5 \%)\end{array}$ & $\begin{array}{c}83,0 \pm 24,0^{*} \\
(\downarrow 32,3 \%)\end{array}$ \\
\hline & Gato & $212,0 \pm 18,3$ & $\begin{array}{c}135,5 \pm 32,1^{*} \\
(\downarrow 36,1 \%)\end{array}$ & $\begin{array}{c}132,5 \pm 43,0^{*} \\
(\downarrow 37,5 \%)\end{array}$ & $\begin{array}{c}147,5 \pm 44,0^{*} \\
(\downarrow 30,5 \%)\end{array}$ & $\begin{array}{c}150,0 \pm 47,5^{*} \\
(\downarrow 29,3 \%)\end{array}$ & $\begin{array}{c}165,5 \pm 60,2^{*} \\
(\downarrow 22,0 \%)\end{array}$ & $\begin{array}{c}172,5 \pm 51,2^{*} \\
(\downarrow 18,7 \%)\end{array}$ \\
\hline \multirow{2}{*}{ PAS } & Cão & $117,5 \pm 28,5$ & $\begin{array}{c}93,8 \pm 25,0^{*} \\
(\downarrow 20,2 \%)\end{array}$ & $\begin{array}{c}85,0 \pm 10,8^{*} \\
(\downarrow 27,7 \%)\end{array}$ & $\begin{array}{c}88,8 \pm 27,5^{*} \\
(\downarrow 24,5 \%)\end{array}$ & $\begin{array}{c}81,3 \pm 16,4^{*} \\
(\downarrow 30,9 \%)\end{array}$ & $\begin{array}{c}88,8 \pm 13,6^{*} \\
(\downarrow 24,5 \%)\end{array}$ & $\begin{array}{c}91,3 \pm 17,3^{*} \\
(\downarrow 22,3 \%)\end{array}$ \\
\hline & Gato & $171,3 \pm 33,3$ & $\begin{array}{c}133,8 \pm 27,4^{*} \\
(\downarrow 21,9 \%)\end{array}$ & $\begin{array}{c}137,5 \pm 27,3^{*} \\
(\downarrow 19,8 \%)\end{array}$ & $\begin{array}{c}147,5 \pm 44,0^{*} \\
(\downarrow 30,5 \%)\end{array}$ & $\begin{array}{c}133,8 \pm 19,3^{*} \\
(\downarrow 21,9 \%)\end{array}$ & $\begin{array}{c}135,5 \pm 24,5^{*} \\
(\downarrow 22,0 \%)\end{array}$ & $\begin{array}{c}172,5 \pm 51,2^{*} \\
(\downarrow 18,7 \%)\end{array}$ \\
\hline \multirow{2}{*}{ FR } & Cão & $37,6 \pm 13,1$ & $\begin{array}{r}22,0 \pm 3,0^{*} \\
(\downarrow 41,5 \%)\end{array}$ & $\begin{array}{r}17,8 \pm 5,1^{*} \\
(\downarrow 52,7 \%)\end{array}$ & $\begin{array}{r}17,3 \pm 5,5^{*} \\
(\downarrow 54,9 \%)\end{array}$ & $\begin{array}{c}18,3 \pm 5,6^{*} \\
(\downarrow 51,4 \%)\end{array}$ & $\begin{array}{r}24,0 \pm 6,0 * \\
(\downarrow 36,2 \%)\end{array}$ & $\begin{array}{c}91,3 \pm 17,3^{*} \\
(\downarrow 22,3 \%)\end{array}$ \\
\hline & Gato & $54,1 \pm 14,9$ & $\begin{array}{c}53,5 \pm 15,4^{*} \\
(\downarrow 1,2 \%) \\
\end{array}$ & $\begin{array}{c}50,5 \pm 13,8 * \\
(\downarrow 6,7 \%) \\
\end{array}$ & $\begin{array}{c}42,6 \pm 13,2^{*} \\
(\downarrow 21,3 \%)\end{array}$ & $\begin{array}{c}36,1 \pm 8,8^{*} \\
(\downarrow 33,3 \%)\end{array}$ & $\begin{array}{c}36,0 \pm 12,6^{*} \\
(\downarrow 33,5 \%)\end{array}$ & $\begin{array}{c}32,5 \pm 8,9 * \\
(\downarrow 40,0 \%)\end{array}$ \\
\hline \multirow{2}{*}{$\mathrm{DP}$} & Cão & $1(1 ; 1)$ & $2^{*}(2 ; 2)$ & $2^{*}(1 ; 2)$ & $1(1 ; 2)$ & $1(1 ; 2)$ & $1(1 ; 2)$ & $1(1 ; 1)$ \\
\hline & Gato & $1(1 ; 1)$ & $2^{*}(2 ; 2)$ & $2^{*}(1 ; 2)$ & $2 *(1 ; 2)$ & $2^{*}(1 ; 2)$ & $2^{*}(1 ; 2)$ & $1,5^{*}(1 ; 2)$ \\
\hline
\end{tabular}

cães: $n=8$; gatos: $n=8$

Valores de referência cães ${ }^{30}: 70-160 \mathrm{bpm}$ (FC); $100-160 \mathrm{mmHg}$ (PAS); $10-30 \mathrm{mpm}$ (FR); Temperatura: $37,8-39,3^{\circ} \mathrm{C}$

Valores de referência gatos ${ }^{30}: 120-240 \mathrm{bpm}$ (FC); $120-170 \mathrm{mmHg}$ (PAS); $20-50 \mathrm{mpm}$ (FR); Temperatura: $38,1-39,2^{\circ} \mathrm{C}$

$\downarrow=$ percentagem de redução em relação ao T0

* diferença significativa $(\mathrm{p}<0,05)$ nos diverso s mom entos em relação ao T0

cardiorrespiratórias induzidas pelo amitraz foi mais significativa nos cães (Tabela 1). A freqüência cardíaca reduziu 51,5\% já nos primeiros 30 minutos após a administração do amitraz, enquanto nos gatos a redução foi de $36,1 \%$. Até o final do experimento a redução da FC nos cães foi de $32,3 \%$ e nos gatos de 18,7\%. Em ambas as espécies, observou-se diminuição da PAS, sendo que a redução foi de $22,3 \%$ nos cães e de 18,3\% nos gatos no T360. Sabe-se que a bradicardia ocorre após a administração do amitraz devido à ativação de receptores $\alpha_{2}$ présinápticos centrais ${ }^{17}$, diminuindo a liberação de dopamina e noradrenalina, reduzindo o tônus simpático, mas também à ação periférica no controle do tônus vascular. ${ }^{18,19}$

As arritmias induzidas com a administração de amitraz estão descritas na tabela 2. Os cães apresentaram maior número de arritmias quando comparado com os gatos. Trinta minutos após a administração do amitraz vários cães apresentaram bloqueio átrio-ventricular (AV) de $1^{0}$ grau, 60 minutos após parada sinusal, 120 e 180 minutos após bradicardia sinusal e 240 minutos após novamente bloqueio AV $1^{0}$ grau. Os gatos só apresentaram alterações significativas, arritmia sinusal, no T180. Sabese que os agonistas $\alpha_{2}$-adrenérgicos

Tabela 2 - Medianas (P25;P75) do ritmo cardíaco em cães e gatos intoxicados por amitraz (1 mg/kg, IV)

\begin{tabular}{cccccccc}
\hline \multirow{2}{*}{ Animal } & \multicolumn{7}{c}{ TEMPO (min) } \\
\cline { 2 - 7 } Cão & T0 & T30 & T60 & T120 & T180 & T240 & T360 \\
\hline \multirow{2}{*}{ Gato } & $1(1 ; 2)$ & $4(3 ; 5)^{*}$ & $5(2 ; 4)^{*}$ & $3(4 ; 5)^{*}$ & $3(4 ; 5)^{*}$ & $4(2 ; 5)^{*}$ & $2(4 ; 5)$ \\
& & $1(1 ; 3)$ & $1(1 ; 3)$ & $1(1 ; 4)$ & $2(1 ; 4)^{*}$ & $1(1 ; 3)$ & $1(1 ; 3)$ \\
\hline
\end{tabular}

As medianas representam os seguintes ritmos cardiacos: (1) sinusal; (2) anitmia sinusal; (3)bradicardia sinusal; (4) bloqueio AV $1^{\circ}$ grau;

(5) parada sinusal. cães: $\mathbf{n}=8$; gatos: $n=8$ 
provocam diminuição da freqüência cardíaca e da pressão arterial, devido à redução do tônus simpático e ao aumento do tônus vagal, induzindo bradicardia sinusal, bloqueio AV de primeiro e segundo graus e, mais raramente de terceiro grau com pulsações de escape..$^{19}$ A bradicardia tende a aumentar a incidência de arritmia sinusal e o aumento do tônus vagal pode induzir ao aparecimento de parada sinusal, ou seja, pausa no ritmo sinusal que é maior que 2 intervalos R-R precedentes. ${ }^{20}$

A diminuição significante $\mathrm{da}$ freqüência respiratória foi observada em cães a partir de T30 (Tabela 1), enquanto em gatos, a partir de T120. Sabe-se que a depressão respiratória induzida pelo amitraz ocorre devido à ação central $\alpha_{2}$-adrenérgica deste acaricida. ${ }^{11}$ Nos gatos os valores de freqüência respiratória relativamente mais altos que os cães nos primeiros momentos do experimento, talvez possam ser explicados pelo estresse inicial induzido nesses animais pela contenção e manuseio realizados, que podem produzir respostas típicas do hormônio adrenocortical do estresse. $^{21}$

Os gatos apresentaram midríase induzida pelo amitraz por mais tempo do que os cães (Tabela 1). A midríase nos gatos persistiu até o T240, enquanto nos cães somente até o T60. Sabe-se que o amitraz causa midríase dose-dependente mediada por $\alpha_{2}$-adrenoceptores pós-sinápticos. ${ }^{22}$

O TMRS foi significativamente diferente entre os cães e gatos após a administração de amitraz. Nos cães o TMRS foi de $149,0 \pm 65,0 \mathrm{~min}$, enquanto nos gatos foi de $175,0 \pm 70,7 \mathrm{~min}$. Uma das principais características da intoxicação por amitraz é a sedação induzida devido ao agonismo $\alpha_{2}$ central. ${ }^{23}$

As alterações endócrinas induzidas pelo amitraz estão descritas na tabela 3. Os gatos apresentaram um pico de hiperglicemia no T60 correspondendo a um aumento de 95,3\%. Nos cães o pico ocorreu no T240 (aumento de 61,8\%). A insulina foi inversamente proporcional à curva de glicose de maneira eqüitativa em ambas as espécies. O mecanismo de ação do amitraz e de seu metabólito ativo BTS 27271 na indução da hiperglicemia ocorre por meio da inibição da secreção de insulina ${ }^{24}$, mediada por receptores $\alpha_{2}$-adrenérgicos, possivelmente pela inibição da adenilciclase, esta mediada por proteínas G PTX-sensitivas ${ }^{25}$. O subtipo de receptor $\alpha_{2}$-adrenérgico envolvido nesta ação é o $\alpha_{2 \mathrm{D}}$ localizado nas ilhotas pancreáticas. ${ }^{26}$ Cães e gatos diabéticos geralmente apresentam curva glicêmica diferenciada. ${ }^{27}$ Gatos não-diabéticos saudáveis podem apresentar hiperglicemia por estresse ${ }^{28}$ o que também poderia colaborar para valores diferenciados nesse caso.

Tabela 3 -Médias e desvios padrões dos valores obtidos de glicose, insulina e cortisol em cães e gatos intoxicados por amitraz $(1 \mathrm{mg} / \mathrm{kg}$, IV)

\begin{tabular}{|c|c|c|c|c|c|}
\hline \multirow{2}{*}{ Variáveis } & \multirow{2}{*}{ Animal } & \multicolumn{4}{|c|}{ Tempo (min) } \\
\hline & & T0 & T60 & T240 & T480 \\
\hline \multirow{2}{*}{$\begin{array}{l}\text { glicose } \\
(\mathrm{mg} / \mathrm{dL})\end{array}$} & Cão & $81,8 \pm 13,4$ & $\begin{array}{c}101,5 \pm 25,2 \\
(\uparrow 24,0 \%)\end{array}$ & $\begin{array}{c}132,4 \pm 68,7^{*} \\
(\uparrow 61,8 \%)\end{array}$ & $\begin{array}{c}87,6 \pm 21,1 \\
(\uparrow 7,0 \%)\end{array}$ \\
\hline & Gato & $85,8 \pm 23,3$ & $\begin{array}{c}167,5 \pm 52,9^{*} \\
(\uparrow 95,5 \%) \\
\end{array}$ & $\begin{array}{c}136,7 \pm 42,0 * \\
(\uparrow 59,3 \%) \\
\end{array}$ & $\begin{array}{c}103,7 \pm 23,7 * \\
(\uparrow 20,8 \%) \\
\end{array}$ \\
\hline \multirow{2}{*}{$\begin{array}{l}\text { insulina } \\
(\mu \mathbf{U} / \mathbf{m L})\end{array}$} & Cão & $0,90 \pm 0,88$ & $\begin{array}{c}0,26 \pm 0,10^{*} \\
(\downarrow 71,2 \%)\end{array}$ & $\begin{array}{c}0,29 \pm 0,11^{*} \\
(\downarrow 67,8 \%)\end{array}$ & $\begin{array}{l}1,91 \pm 0,93 * \\
(\uparrow 112,2 \%)\end{array}$ \\
\hline & Gato & $0,81 \pm 0,58$ & $\begin{array}{c}0,30 \pm 0,28^{*} \\
(\downarrow 63,0 \%)\end{array}$ & $\begin{array}{c}1,04 \pm 1,19 * \\
(\downarrow 28,3 \%)\end{array}$ & $\begin{array}{c}0,30 \pm 0,21 * \\
(\downarrow 63,0 \%)\end{array}$ \\
\hline \multirow{2}{*}{$\begin{array}{l}\text { cortisol } \\
(\mu \mathrm{U} / \mathrm{dL})\end{array}$} & Cão & $0,90 \pm 0,66$ & $\begin{array}{c}0,49 \pm 0,13 * \\
(\downarrow 45,5 \%)\end{array}$ & $\begin{array}{c}0,50 \pm 0,10 * \\
(\downarrow 44,5 \%)\end{array}$ & $\begin{array}{c}0,83 \pm 0,24 \\
(\downarrow 7,8 \%)\end{array}$ \\
\hline & Gato & $2,90 \pm 1,55$ & $\begin{array}{c}1,80 \pm 0,89 * \\
(\downarrow 38,0 \%)\end{array}$ & $\begin{array}{c}2,49 \pm 1,26 \\
(\downarrow 14,2 \%)\end{array}$ & $\begin{array}{c}2,91 \pm 1,26 \\
(\uparrow 0,3 \%)\end{array}$ \\
\hline
\end{tabular}

* diferença significativa $(\mathrm{p}<0,05)$ em relação ao TO

$\downarrow=$ percentagem de redução em relação ao $\mathrm{T} 0 ; \uparrow=$ percentagem de aumento em relação ao $\mathrm{T} 0$ 
Os níveis de cortisol foram mais baixos nos cães, havendo nos tempos T60, T240 e T480 respectivamente diminuições de $45,5 \%, 44,5 \%$ e 7,8\%. Nos gatos, no tempo T60, a diminuição foi de $38,0 \%$. A diminuição dos níveis de cortisol ocorreu provavelmente devido a administração do amitraz causar depressão do sistema nervoso central (SNC), por estimulação de $\alpha_{2}$-adrenoceptores pré-sinápticos, resultando numa diminuição do efluxo simpático do SNC e diminuição das catecolaminas e de outras substâncias relacionadas ao estresse. ${ }^{21,25}$ O cortisol é considerado o hormônio do estresse, portanto, níveis plasmáticos de cortisol, insulina e glicose podem estar alterados em animais estressados. ${ }^{21}$ Os gatos são mais susceptíveis às alterações induzidas pelo estresse do que os cães ${ }^{28,29}$, o que poderia explicar essas diferenças observadas nos níveis de cortisol induzidos pela intoxicação por amitraz .

\section{Conclusões}

Cães e gatos, de uma maneira geral, apresentam os mesmos sinais clínicos da intoxicação por amitraz, ou seja, principalmente sedação, hipotermia, bradicardia, bradiarritmias, hipotensão, bradipnéia, midríase e hiperglicemia transitória, porém com intensidade diferente.

Aparentemente os gatos são mais sensíveis às ações centrais do amitraz apresentando uma hipotermia mais acentuada, além de sedação e persistência da midríase mais prolongada. A hiperglicemia induzida pelo amitraz em gatos também foi diferenciada daquela observada em cães, sendo que os gatos apresentaram uma curva glicêmica com pico maior e mais precoce.

Os cães foram sensíveis às ações centrais do amitraz, mas possivelmente também às ações periféricas, o que pode explicar a maior sensibilidade às alterações cardiorrespiratórias induzidas com esta intoxicação, como bradicardia e bradipnéia mais acentuadas, e uma maior ocorrência de bradiarritmias. Considerando as alterações endócrinas, os cães foram mais sensíveis à diminuição de cortisol, o que pode ser devido a essa espécie ser menos susceptível ao estresse quando comparada aos gatos.

Essas observações contribuem com mais subsídios para o entendimento desta intoxicação nestas duas espécies, o que pode ser útil no direcionamento do tratamento e prognóstico da intoxicação por amitraz em cães e gatos.

Auxílio financeiro da Pós-Graduação da Universidade do Oeste Paulista, (UNOESTE).

\section{Comparative study of experimental amitraz intoxication between dogs and cats}

\section{Abstract}

The objective of the present work was to study the characteristics in common and the differences observed in the intoxication by amitraz $1 \mathrm{mg} / \mathrm{kg}, \mathrm{IV}$, in dogs and cats. The main clinical signs observed in common were sedation, hypothermia, bradycardia, bradyarrhytmia, hypotension, bradypnea, mydriasis and transitory hyperglycemia, however the intensity of these signs was different among the species. The hypothermia was more accentuated in cats than in dogs. The dogs were more sensitive to the cardiorespiratory alterations presenting more significant decrease in the heart and breathing frequency, besides occurrence of larger bradyarrhytmia number. The cats presented more prolong mydriasis than the dogs. It was observed hyperglycemia and transitory hypoinsulinemia and transitory decrease of the plasmatic
Key words:

Amitraz.

Intoxication.

Dogs.

Cats.

Antiparasitic agents. 
levels of cortisol, however the cats presented a hyperglycemia pick more accentuated and more precocious than the dogs, and regarding the plasmatic levels of cortisol, the dogs presented a decrease more accentuated than the cats. In the medium time of return of the sedation smaller in cat. These results demonstrated that the amitraz intoxication between dogs and cats is very similar, however the cats showed more sensibility to the hypothermia and hyperglycemia induction, besides mydriasis and the medium time of return of sedation more prolong, while the dogs presented a decrease more accentuated in the cardiorespiratory parameters and of the plasmatic levels of cortisol than the cats.

\section{Referências}

1 ANDRADE, S. F.; SAKATE, M. Intoxicação por amitraz. Veterinária Notícias, v. 10, n. 1, p. 101-109, 2004.

2 HSU, W. H. Antiparasitic Agents. In: AHRENS, F. A . Pharmacology, Baltimore: Willians \& Wilkins, 1996. p. $243-260$

3 HUGNET, C.; BURONROSSE, F.; PINEAU, X.; CADORE, J. L.; LORGUE, G.; BERNY, P. J. Toxicity and kinetic of amitraz in dogs. American Journal of Veterinary Research, v. 57, n. 10, p. 1506-1510, 1996.

4 ANDRADE, S. F.; SANCHES, O.; TOSTES, R. A. Relato de cinco casos de intoxicação por amitraz em cães e gatos. Clínica Veterinária, v. 9, n. 53, p. 38-42, 2004.

5 LARSSON, C. E.; GONÇALVES, M. A. Aspectos clínicos da terapia da demodicose canina generalizada com diamidina (amitraz). Cães e Gatos, v. 1, p. 6-10, 1986.

6 COWAN, L. A.; CAMPBELL, K. Generalized demodicosis in a cat responsive to amitraz. Journal of American Veterinary Medical Association, v. 192, n. 10, p. 1442-1444, 1988.

7 CHESNEY, C. J. Demodicosis in the cat. Journal of Small Animal Practice, n. 30, p. 689-695, 1989.

8 XAVIER, F. G.; KOGIKA, M. M.; SPINOSA, H. S. Common causes of poisoning in dogs and cats in a Brazilian Veterinary Teaching Hospital from 1998 to 2000. Veterinary and Human Toxicology, v. 44, n. 2, p. 115-116, 2002.

9 GUNARATNAN, P.; WILKINSON, G. T.; SEAWRIGHT, A. A. A study of amitraz toxicity in cats. Australian Veterinary Journal, v. 60, n. 9, p. 278-279, 1983.

10 SOLI, N. E.; BRASETH, T. A. Ectodex poisoning in a cat reversed with Antisedan. Norsk-Veterinaertidsskrift, v. 104, n. 2, p. 125-126, 1992.

11 CULLEN, L. K.; REYNOLDSON, J. A. Central and peripheral alpha-adrenoceptor actions of amitraz in the dog. Journal of Veterinary Pharmacology Therapeutics, v. 13 , n. 1, p. 86-92, 1990.

12 ANDRADE, S. F.; SAKATE, M. The comparative efficacy of yohimbine and atipamezole to treat amitraz intoxication in dogs. Veterinary and Human Toxicology, v. 45, n. 3, p. 124-127, 2003.

13 ANDRADE, S. F.; SAKATE, M.; CROCCl, A. J. Effects of yohimbine and atipamezole on plasmatic glucose concentration and blood gas analysis in dogs intoxicated with Triatox ${ }^{\oplus}$. ARS Veterinaria, v. 21, p. 121-128, 2005. Suplemento.

14 ANDRADE, S. F.; SAKATE, M.; LAPOSY, C. B.; SANGIORGIO, F. Yohimbine and atipamezole on the treatment of experimentally induced amitraz intoxication in cats. International Journal of Applied Research in Veterinary Medicine, v. 4, n. 3, p. 200208, 2006.

15 STEEL, R. G. B.; TORRIE, J. H. Principles and procedures of statistics. 2 ed. New York: McGrawHill, 1980. 633 p.

16 MICHELL, A. R. Physiology. In: HALL, L. W.; TAYLOR, P. M. Anaesthesia of the cat. 1. ed. London: Bailliére Tindall, 1994. p. 33-34.

17 HSU, W. H.; LU, Z.-X.; HEMBROUGH, F. B. Effect of amitraz on heart rate and aortic blood pressure in conscious dogs: Influence of atropine, prazosin, tolazoline, and yohimbine. Toxicology Applied Pharmacology, v. 84, n. 2, p. 418-422, 1986.

18 RUFFOLO Jr, R. R.; NICHOLS, A. J.; STADEL, J. M.; HIEBLE, J. P. Pharmacologic and therapeutic applications of alpha 2-adrenoceptor subtypes. Annual Review of Pharmacology and Toxicology, n. 33, p. 243-279, 1993.

19 HAYASHI, Y.; MAZE, M. Alpha ${ }_{2}$ adrenoceptor agonists and anaesthesia. British Journal of Anaesthesia v. 71, n. 1, p. 108-118, 1993.

20 EDWARDS, N. J. ECG - manual for the veterinary technician. 1 ed. Philadelphia: W. B. Saunders, 1996. 191 p.

21 NOBLE, R. E. Diagnosis of stress. Metabolism, v. 51, n. 6, p. 37-39, 2002.

22 HSU, W. H.; KAKUK, T. J. Effect of amitraz and chlordimeform on heart rate and pupil diameter in rats: Mediates by $\alpha_{2}$-adrenoceptors. Toxicology and Applied Pharmacology, v. 73, n. 3, p. 411-415, 1984. 
$23 \mathrm{HSU}$, W. H.; HOPPER, D. L. Effect of yohimbine on amitraz-induced CNS depression and bradycardia in dogs. Journal of Toxicology Environmental Health, v. 18, n. 3, p. 423-429, 1986.

$24 \mathrm{HSU}$, W. H.; SCHAFFER, D. D. Effects of topical application of amitraz on plasma glucose and insulin concentrations in dogs. American Journal of Veterinary Research, v. 49, n. 1, p. 130-131, 1988.

25 CHEN, T. H.; HSU, W. H. Inhibition of insulin release by a formamidine pesticide amitraz and its metabolites in a rat beta-cell line: an action mediated by alpha-2 adrenoceptors, a GTP-binding protein and decrease in cyclic AMP. Journal of Pharmacology Experimental Therapeutics, v. 273, n. 3, p. 1240-1245, 1994.

26 ABU-BASHA, E. A.; YIBCHOK-ANUN, S.; HSU, $\mathrm{H}$.; HSU, W. H. Effects of the pesticide amitraz and its metabolite BTS 27271 on insulin and glucagon secretion from the perfused rat pancreas: involvement of $\alpha_{2 D^{-}}$ adrenergic receptors. Metabolism, v. 48, n. 11, p. 14611469, 1999.

27 NELSON, R. W. Oral medications for treating diabetes mellitus in dogs and cats.Journal of Small Animal Practice, v. 41, n. 11, p. 486-490, 2000.

28 CRENSHAW, K. L.; PETERSON, M. E.; HEEB, L. A. serum fructosamine concentration as an index of glycemia in cats with diabetes mellitus and stress hyperglycemia. Journal of Veterinary Internal Medicine, v. 10, n. 6, p. 943-949, 1996.

29 KOJIMA, K.; MOHAMED, S.; FUJIMARU, Y.; MORI, Y.; KANAME, H.; SUMIDA, Y; KINUKAWA, $\mathrm{N}$.; TASHIRO, N. Effects of both the emotional behavior and feeding conditions or the circulation plasma volume and plasma glucose levels in cats. Autonomic Neuroscience, v. 86, n. 1-2, p. 58-64, 2000.

30 BISTNER, S. I.; FORD, R. B. Manual de procedimentos veterinários e tratamento de emergências. 7. ed. São Paulo: Roca, 2002. 934 p. 